\title{
Outcomes After Urgent Thyroidectomy Following Rapid Control of Thyrotoxicosis in Graves' Disease are Similar to Those After Elective Surgery in Well-Controlled Disease
}

\author{
Adibah Ali $^{1} \cdot$ Miguel Debono $^{2} \cdot$ Sabapathy P. Balasubramanian ${ }^{1,3}$
}

Published online: 12 August 2019

(C) The Author(s) 2019

\begin{abstract}
Background Surgery for Graves' disease (GD) is usually performed after adequate control with medical treatment. Occasionally, rapid pre-operative optimization is required. The primary objective was to compare the outcomes of patients undergoing elective surgery for well-controlled GD with those undergoing rapid pre-operative treatment. We also propose a formal treatment protocol for future use.

Methods A retrospective cohort study in a tertiary referral centre included 247 patients with well-controlled GD undergoing elective surgery and 19 patients with poorly controlled disease undergoing surgery after rapid optimization. The latter group did not respond well to thionamides (carbimazole and/or propylthiouracil) or had intolerance or side effects to thionamides and were treated with a range of non-thionamide drugs, including Lugol's iodine, cholestyramine, beta blockers and steroids (with or without thionamides), and closely monitored for 1-2 weeks before surgery. Outcome measures included thyroid storm, hypoparathyroidism and recurrent laryngeal nerve palsy.

Results In total, 266 patients with male-to-female ratio of 1:6 and median (interquartile range) age of 39 (31-51) were included. Overall, long-term recurrent laryngeal palsy and hypoparathyroidism occurred in $1(0.38 \%)$ and 13 $(4.9 \%)$ patients, respectively. No patient had thyroid storm. There was no significant difference in hypoparathyroidism $(p=1)$, vocal cord palsy $(p=0.803)$ and post-operative bleeding $(p=0.362)$, between elective surgery and rapid optimization groups.

Conclusion Rapid pre-operative treatment is effective, safe and is associated with similar outcomes compared to usual treatment. A rapid pre-operative optimization protocol is proposed.
\end{abstract}

Electronic supplementary material The online version of this article (https://doi.org/10.1007/s00268-019-05125-5) contains supplementary material, which is available to authorized users.

Sabapathy P. Balasubramanian

s.p.balasubramanian@sheffield.ac.uk

1 Endocrine Surgery Unit, Sheffield Teaching Hospitals NHS Foundation Trust, F8, Ward F2, Royal Hallamshire Hospital, Beech Hill Road, Sheffield S10 2JF, UK

2 Department of Endocrinology, Sheffield Teaching Hospitals NHS Foundation Trust, Sheffield, UK

3 Department of Oncology and Metabolism, University of Sheffield, Sheffield, UK

\section{Introduction}

Graves' disease (GD) represents $50-80 \%$ of all patients with hyperthyroidism [1]. It occurs more commonly in women, with adjusted female-to-male ratio of 3.9:1 [2].

The main goal of treatment is to normalize thyroid hormone levels and achieve remission. The three current modalities for treatment of GD include anti-thyroid drugs (ATD), radioiodine ablation (RIA) and surgery. ATD treatment is the initial standard treatment, usually lasting for between 6 and 18 months [3]. It induces remission in over two-thirds of patients, of which over a third (36.7\%) relapse in the short to medium term [2]. Definitive 
treatment with either RIA or surgery is considered in relapsed or uncontrolled GD. Surgery is preferred in patients who are pregnant, unsuitable for RIA or those with severe eye disease or large goitres [4]. The main complications of thyroid surgery are laryngeal nerve injury and hypoparathyroidism (2-5\%) [5]. Uncommon complications include post-operative bleeding and infection.

The risk of perioperative thyroid storm is reduced by adequate pre-operative optimization with appropriate medical treatment [6]. Different strategies have been proposed to facilitate this [7-9]. In patients with uncontrolled disease and those who need urgent surgery, rapid pre-operative preparation may be necessary. Panzer et al. [10] demonstrated the effectiveness of a rapid preparation protocol $(n=17)$ with $500 \mathrm{mg}$ iopanoic acid twice a day, $1 \mathrm{mg}$ dexamethasone twice a day along with beta blockers and thionamides over 7 days. This is similar to other studies on the safety and effectiveness of regimens including ATD in addition to either sodium iopodate or iopanoic acid [7, 8] or combinations of steroids, iopanoic acid and beta blockers [8, 9]. These studies appear to have used a rigid protocol irrespective of the severity of thyrotoxicosis and previous tolerance to ATD treatments. Most studies did not have a control group $[8,9,11]$ except one which had a very small number of control patients undergoing surgery for well-controlled disease [7].

Surgery for GD is thought to be associated with an increased risk of complications compared to other thyroid conditions [12-14]. There is also a concern that patients with poorly controlled disease have an increased risk of complications [15], and post-operative hungry bone syndrome may compound the effects of post-operative hypoparathyroidism [16].

The aim of this study is to describe the clinical outcomes of patients undergoing urgent surgery for Graves' disease following rapid optimization and compare these to patients undergoing elective surgery for well-controlled disease. We also describe the effectiveness of treatment regimens used in the latter group of patients and propose a formal protocol for future use.

\section{Methods}

This is a retrospective cohort study of consecutive patients who underwent surgery for GD in one unit over a 6-year period (January 2010 to December 2016). Data on demographics, surgical and perioperative details, and clinical outcomes were collected from electronic records and recorded in an Excel spreadsheet.

Laryngoscopy, anaesthetic assessment, thyroid and calcium biochemistry were routinely performed before surgery. For the purpose of this study, patients with controlled
GD are those with normal or mildly elevated thyroid hormone levels $($ FT4 $<30 \mathrm{pmol} / \mathrm{L}$ and FT3 $<10 \mathrm{pmol} / \mathrm{L})$ and clinically euthyroid with outpatient medical treatment and underwent thyroidectomy as a planned elective procedure. The indication for surgery included one of the following-relapsed Graves' disease needing further medical treatment, associated large goitre, significant thyroid eye disease or patient wish for definitive treatment [2]. These patients were usually on ATD (carbimazole or propylthiouracil) either as sole treatment (dose titration regimen) or on ATD and thyroxine (block and replace regime). Most patients were prescribed Lugol's iodine at dose of $0.3 \mathrm{~mL}$ three times a day for 10 days before surgery. Patients with poorly controlled GD and/or those who required rapid optimization were either treated as frequent outpatient visits or as inpatients for rapid control of thyrotoxicosis prior to surgery using a combination of different drugs. These patients were either intolerant or responded poorly to anti-thyroid drugs and needed urgent definitive treatment (reasons included significant thyroid eye disease, awaiting treatment for other medical conditions or patient choice). These patients were defined as having either FT4 $>30 \mathrm{pmol} / \mathrm{L}, \mathrm{FT} 3>10 \mathrm{pmol} / \mathrm{L}$ or had thyroid hormone levels persistently above the normal range with clinical features of hyperthyroidism (as judged by the treating clinician). Treatment included a combination of ATD, Lugol's iodine, cholestyramine, beta blockers and steroids, continued up to the day of surgery.

After surgery, patients were started on $1.6 \mathrm{mcg} / \mathrm{kg}$ of thyroxine. Routine prophylactic calcium or vitamin D supplements were not used. Vitamin D deficiency was treated if detected-either before or immediately after surgery. Post-operative hypocalcaemia and hypoparathyroidism were tested for and treated in accordance with a standard protocol [17]. All patients were routinely offered laryngoscopy after surgery. Outcomes assessed included the occurrence of thyroid storm, temporary and long-term hypocalcaemia and/or hypoparathyroidism, recurrent laryngeal nerve injury, length of post-operative hospital stay and readmission within 30 days of surgery. Thyroid storm was defined as the occurrence of thyrotoxicosis and at least one neurological manifestation and one of fever, tachycardia, congestive heart failure, gastrointestinal or hepatic features [18]. Temporary hypocalcaemia was defined as adjusted calcium level below $2.1 \mathrm{mmol} / \mathrm{L}$ on the first post-operative day. Patients were considered to have long-term hypoparathyroidism if they required treatment for over 6 months. Recurrent laryngeal nerve palsy was identified at laryngoscopy, and patients were considered to have long-term palsy if this lasted for over 6 months. The reference ranges included $0.27-4.2 \mathrm{mIU} / \mathrm{L}$ for $\mathrm{TSH}$, 12-22 pmol/L for FT4, 3.1-6.8 pmol/L for FT3, 2.1-2.6 mmol/L for calcium and 1.6-6.9 $\mathrm{pmol} / \mathrm{L}$ for iPTH. 
The data were exported to SPSS for analysis. Data were described as frequencies and/or percentages (for categorical data); mean and standard deviation for continuous, normally distributed data; and median (and interquartile range) for continuous data that were not normally distributed. The Pearson Chi-square test with continuity correction or Fisher's exact test was used to determine associations and differences in categorical data sets. The Student's $t$ test or the Mann-Whitney $U$ test was used for comparisons of continuous data depending on whether the data were normally distributed or not. A significance value of $p<0.05$ was used.

The study was registered with the R\&D Department in Sheffield Teaching Hospitals (STH 20132). As this was an observational study, a formal ethics application and individual patient consent were not deemed necessary. Patient confidentiality and privacy was maintained during data collection, and the data were anonymized before analysis and reporting.

\section{Results}

Surgery was performed in 266 patients with a male-tofemale ratio of 1:6. The median (25th-75th centile) age at surgery was 39 (31-51) years. Five (1.9\%) patients had a documented history of radioiodine ablation. All patients (except one who underwent subtotal resection) underwent total or near total thyroidectomy. Four consultants were involved in either performing the surgery or supervising trainees. Of 266 patients, 247 (92.9\%) patients were well controlled and had elective surgery. Nineteen (7.1\%) patients were considered to be poorly controlled in an outpatient setting and/or required rapid optimization prior to surgery. Of these, 14 were treated as inpatients while 5 patients were monitored daily as outpatient. Five (26\%) patients were intolerant to anti-thyroid drugs and were managed with other agents. Figure 1 shows the treatment regimens administered in the poorly controlled group. Five patients required dose adjustments (3 patients required increased PTU; 2 patients required increased propranolol). Steroids were administered in 2 patients about 2-3 days prior to surgery. Two patients were intolerant to cholestyramine. The baseline characteristics of patients are displayed in Table 1.

The median (25th-75th centile) FT4 and FT3 levels at the time of listing for surgery were $40(31-75) \mathrm{pmol} / \mathrm{L}$ and 15 (10-21) pmol/L, respectively, for the rapidly optimized group. This was higher (Fig. 2) than the well-controlled group [15 (12-18) pmol/L and 5 (4-6) pmol/L for FT4 and FT3, respectively]. There was a downward trend in hormone levels in all patients after commencing rapid treatment (suppl. Figures 1 and 2). The majority of patients demonstrated a reducing trend in FT4 levels within 3 days after rapid treatment was initiated. Only 8 (42\%) and 10 (53\%) patients had normal free T4 and T3 levels, respectively, prior to surgery as displayed in Table 2 . Six patients (32\%) had both free T4 and T3 levels slightly elevated at the time of surgery.

Parathyroid auto-transplantation was done on $9.8 \%$ $(n=24)$ patients in the well-controlled group and none in the poorly controlled group ( $p=0.2$, Fisher's exact test). At least one parathyroid gland was found on histology in $6.9 \%(n=17)$ and $10.5 \%(n=2)$ of the well- and poorly controlled patients, respectively ( $p=0.6$, Fisher's exact test).

No patient in the cohort had thyroid storm. Sixty-six (24.9\%) patients had low post-op day 1 adjusted calcium; $62(23.6 \%)$ required treatment for hypocalcaemia; and 13 (4.9\%) had long-term hypoparathyroidism. There was no significant difference in temporary or long-term hypoparathyroidism rates and rates of reoperation for bleeding between consultants. Eighteen (7\%) patients had post-operative vocal cord palsy and/or significant voice
Fig. 1 Management regimens used in rapidly optimization of poorly controlled Graves' disease. ATD anti-thyroid drugs, $C M Z$ carbimazole, $P T U$ propylthiouracil, Chole cholestyramine, $B B$ beta blocker, $V R P$ verapamil

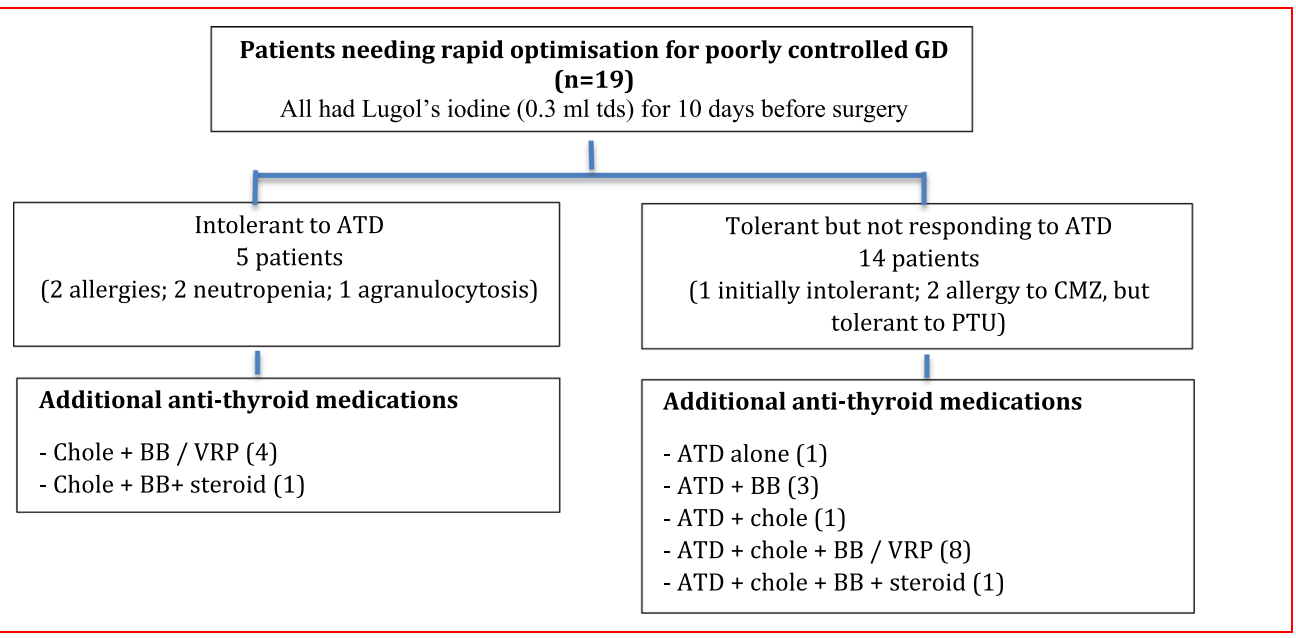


Table 1 Comparison of baseline characteristics in well-controlled, elective versus rapidly optimized patients with Graves' disease

\begin{tabular}{llll}
\hline & Well-controlled, elective $(\%)$ & Rapidly optimized, urgent $(\%)$ & $P$ value \\
\hline M/F ratio & $1: 6.5(n=247)$ & $1: 18(n=19)$ & $33(23-38)$ \\
Median age $(25$ th-75th) & $40(30-51)$ & $0(0)$ & \\
History of RIA $(n=245)$ & $5(2)$ & & \\
Consultants & & $2(11)$ & $0.483^{*}$ \\
A & $77(31)$ & $0(0)$ & $0.004^{* *}$ \\
B & $16(6)$ & $0(0)$ \\
C & $4(2)$ & $17(89)$ \\
D & $150(61)$ & $(n=19)$ \\
& $(n=245)$ & $201(182-218)$ \\
Median duration of surgery (min) & $191(164-221)$ & $2(1-4)$ \\
Median hospital stay after surgery (days) & $1(1-2)$ & $0.355^{* *}$ \\
\hline
\end{tabular}

Bold value indicates significant results

*Fisher's exact test

**Mann-Whitney $U$ test
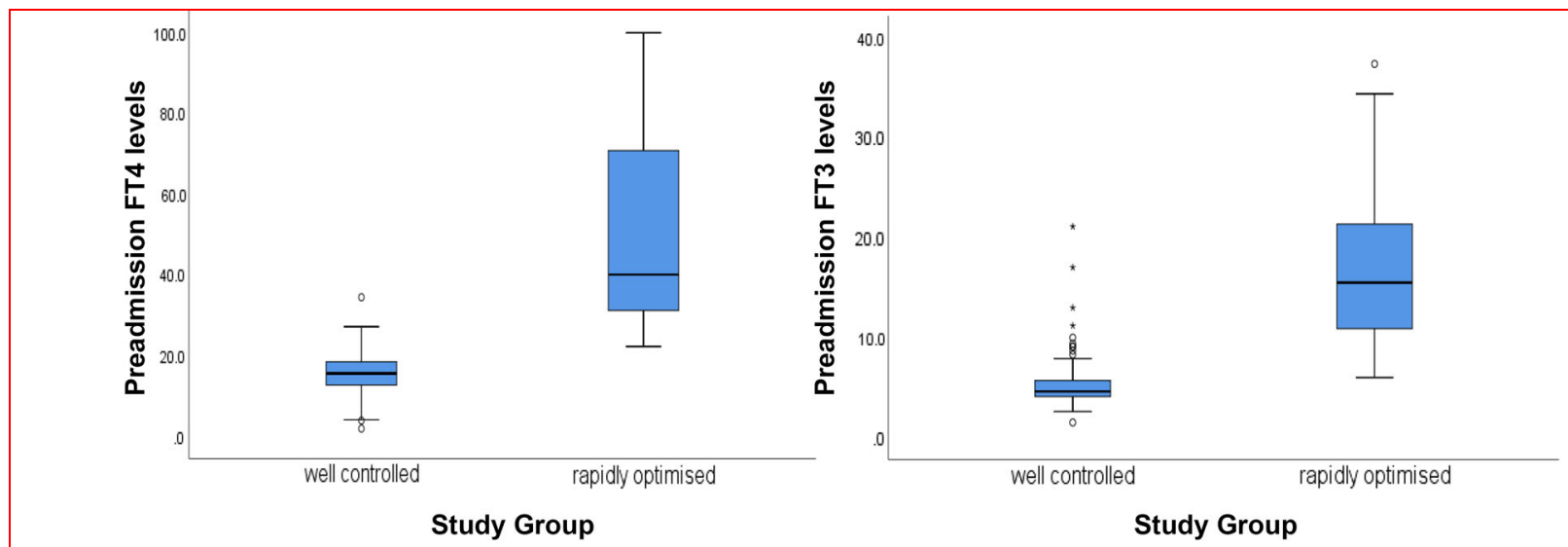

Fig. 2 Pre-admission free T4 and free T3 levels in well-controlled, elective and rapidly optimized, urgent patients with Graves' disease

change. Of these patients, only 1 had a documented persistent cord palsy at 6 months. There was a statistically significant difference in temporary vocal cord paresis (and/ or significant voice problems) between consultants ( $p=0.021$; Fisher's exact test), but not in long-term rates. Six $(2.3 \%)$ patients required surgery for post-operative bleeding. One patient (in the rapidly pre-optimized group) who underwent re-exploration for bleeding after thyroidectomy required admission to ICU for monitoring after surgery, due to difficulty in intubation at the second operation. The comparison of outcomes in the well- and poorly controlled groups is shown in Table 3 .

\section{Discussion}

This study demonstrates that outcomes following elective surgery in well-controlled disease and urgent surgery after rapid pre-operative optimization of thyroid function tests are similar. Based on this experience, we have developed and proposed a protocol to be used by clinicians when managing uncontrolled patients with Graves' disease prior to surgery (Fig. 3).

The indications for surgery in GD vary between centres in Europe, USA and worldwide [19, 20]. In the UK, the majority of consultants preferred surgery over RIA for relapsed Graves' disease, especially in the presence of thyroid eye disease and pregnancy [21]. Other indications for surgery include suspected thyroid malignancy, large thyroid nodule, coexisting primary hyperparathyroidism and a contraindication for ATD and RIA [21-23]. 
Table 2 Comparison of hormonal levels before and after rapid optimization prior to surgery in poorly controlled GD

\begin{tabular}{|c|c|c|c|c|c|c|}
\hline \multirow[t]{2}{*}{ Patients } & \multicolumn{2}{|c|}{ FT4 (pmol/L) } & \multicolumn{2}{|c|}{ FT3 (pmol/L) } & \multicolumn{2}{|c|}{ TSH (mIU/L) } \\
\hline & Before & After & Before & After & Before & After \\
\hline 1 & $>100$ & 30.2 & 35.2 & 6.7 & $<0.02$ & NA \\
\hline 2 & 24.5 & 12.8 & 7.1 & 3.3 & $<0.02$ & $<0.02$ \\
\hline 3 & 24.3 & 8.3 & 23.9 & 7 & $<0.02$ & $<0.02$ \\
\hline 4 & 36.4 & 29.6 & 15.7 & 8.5 & $<0.02$ & $<0.02$ \\
\hline 5 & 37.5 & 29.4 & 11.5 & 8 & $<0.02$ & NA \\
\hline 6 & $>100$ & 47.8 & 10.3 & 7.6 & 0.03 & 0.87 \\
\hline 7 & 62.6 & 25.6 & 37.4 & 5.8 & $<0.02$ & $<0.02$ \\
\hline 8 & 61 & 29.1 & 28.2 & 8.6 & $<0.02$ & $<0.02$ \\
\hline 9 & 79 & 26.4 & 17.4 & 4.8 & $<0.02$ & $<0.02$ \\
\hline 10 & 91.5 & 32.5 & $>50$ & 11.4 & $<0.02$ & $<0.02$ \\
\hline 11 & 91.8 & 21.2 & 39.7 & 6.9 & $<0.02$ & $<0.02$ \\
\hline 12 & $>100$ & 22.5 & $>50$ & 8.6 & $<0.02$ & $<0.02$ \\
\hline 13 & 27.1 & 14.7 & 10.3 & 4.4 & $<0.02$ & $<0.02$ \\
\hline 14 & 30.9 & 11.6 & 9.7 & 2.6 & NA & NA \\
\hline 15 & 42.5 & 11.6 & 15.5 & 2.6 & $<0.02$ & $<0.02$ \\
\hline 16 & $>100$ & 18.3 & 34.4 & 6.4 & $<0.02$ & $<0.02$ \\
\hline 17 & 22.3 & 16.6 & 4 & 3 & $<0.03$ & $<0.03$ \\
\hline 18 & 31.5 & 21.9 & 11.8 & 5.9 & $<0.03$ & $<0.01$ \\
\hline 19 & 27.6 & 15.1 & 12.8 & 4.8 & $<0.03$ & $<0.03$ \\
\hline
\end{tabular}

However, temporary recurrent laryngeal nerve palsy and permanent hypoparathyroidism are reported to be higher after surgery for Graves' compared to surgery for other benign thyroid diseases ( $8.3 \%$ vs. $0 \%$ and $11.5 \%$ vs. $2.5 \%$, respectively) [14].

In this study, $4.9 \% \quad(n=13)$ had long-term hypoparathyroidism and 1 patient had long-term nerve palsy. This is comparable to a large national database study which reported that $12.4 \%$ and $0.9 \%$ had hypoparathyroidism and vocal cord palsy, respectively [12]. However, the hypoparathyroidism and unilateral vocal cord palsy were not clearly defined in this report. In a meta-analysis by Tapash et al. [24], permanent recurrent laryngeal nerve injury occurred in $0.9 \%$ of TT patients and $0.7 \%$ of ST patients, while permanent hypoparathyroidism occurred in $1.6 \%$ of TT patients and $1.0 \%$ of ST patients.

In the well-controlled, elective group, the surgery was performed without rapid optimization in some patients who had high free T3 levels prior to surgery because the patients were euthyroid and free T4 levels were still in normal range (Fig. 2).

In this series, the patients in the rapidly optimized group are predominantly women and significantly younger compared to the well-controlled group (median of 33 vs. 40 years, respectively, $p=0.004$ ). Young age limits the option of RIA as a number of young women are concerned about pregnancy [25]. Furthermore, eye disease (commonly present in rapidly optimized GD) has a higher risk of progression in patients treated with RIA compared to surgery [26, 27]. In addition, Lugol's iodine or iopodate cannot be used in patients undergoing RIA. Therefore, surgery following rapid optimization is often the preferred treatment in many patients with poorly controlled disease.

Preparation for surgery in poorly controlled GD requires close monitoring and dose adjustments. Although some studies suggest that biochemical hyperthyroidism may not increase morbidity [28, 29], these are retrospective studies from high volume centres and may not be generalizable. It is also not clear whether these patients had reducing free T3 and T4 levels prior to surgery. Current guidelines from the American Thyroid Association strongly recommend that patients 'should be rendered euthyroid prior to the procedure' [23].

Rendering patients euthyroid can be achieved by varying combinations of ATD and other drugs (ipodate, iodinated radiographic contrast agent, dexamethasone, B-blocker and cholestyramine). A comparative study had described that the combination of $500 \mathrm{mg}$ iopanoic acid twice a day, $1 \mathrm{mg}$ dexamethasone twice a day, beta blocker and ATD rapidly reduced the T4 and T3 in majority of patients $(80 \%)$ to normal levels in 7 days [10]. In contrast, although the majority of patients in our current study had

Table 3 Comparison of clinical outcomes between well-controlled, elective versus rapidly optimized patients with Graves' disease

\begin{tabular}{llll}
\hline & Well-controlled, elective $(\%)$ & Rapidly optimized, urgent $(\%)$ & $P$ value* \\
\hline Low 'adjusted serum calcium' on day after surgery & $62 / 246(25.2)$ & $4 / 19(21.1)$ & $5 / 19(26.3)$ \\
Post-op hypocalcaemia needing treatment & $57 / 243(23.5)$ & $1 / 19(5.3)$ & 0.790 \\
Long-term (6 months) hypoparathyroidism & $12 / 244(4.9)$ & $0 / 18(0)$ & 0.781 \\
Post-op cord palsy and/or significant voice problem & $18 / 238(7.6)$ & $0 / 19(0)$ & 1 \\
6-month cord check & $1 / 241(0.4)$ & $1 / 19(5.3)$ & 0.624 \\
Post-operative bleeding & $5 / 247(2)$ & 0.803 \\
\hline
\end{tabular}

*Fisher's exact test 
10 days before surgery

Start Lugol's iodine, propanolol $20 \mathrm{mg}$ tds and continue all other meds incl. ATDs if tolerated

7 days before surgery

Attend ward for clerking and bloods incl FT4 \& FT3

FT4 $>30 \mathrm{mmol} / \mathrm{L}$ or FT3 $>10 \mathrm{mmol} / \mathrm{L}$ - add

cholestyramine; increase propanolol dose

Satisfactory FT4 and FT3 - continue meds

5 days before surgery

Review FT4 \& FT3

FT4 $>30 \mathrm{mmol} / \mathrm{L}$ or FT3 $>10 \mathrm{mmol} / \mathrm{L}$ - add dexamethasone; increase propanolol dose

Satisfactory FT4 and FT3 - continue meds

3 days before surgery

Review FT4 \& FT3

FT4 $>30 \mathrm{mmol} / \mathrm{L}$ or FT3 $>10 \mathrm{mmol} / \mathrm{L}$ - increase dexamethasone and propanolol

Satisfactory FT4 and FT3 - continue meds

1 day before surgery

Review FT4 \& FT3

FT4 $>\mathbf{3 0 ~} \mathrm{mmol} / \mathrm{L}$ or FT3 $>\mathbf{1 0} \mathrm{mmol} / \mathrm{L}-$ Discuss

with anaes/endo; consider postponing surgery

Satisfactory FT4 and FT3 - continue meds and

plan for surgery

Fig. 3 Proposed protocol for the rapid optimization of thyroid function prior to surgery in poorly controlled Graves' disease. Notes The proposed starting doses of the medications are Lugol's iodine at $0.3 \mathrm{~mL}$ tds, propranolol $20 \mathrm{mg}$ tds, cholestyramine $4 \mathrm{~g}$ tds and dexamethasone $1 \mathrm{mg}$ bd. The dose of propranolol may be increased up to $80 \mathrm{mg}$ tds and that of dexamethasone to $2 \mathrm{mg}$ bd. For patients on cholestyramine, no other medications should be administered at least $2 \mathrm{~h}$ before and $4 \mathrm{~h}$ after cholestyramine

showed a declining trend in hormone levels 3 days after initiating the treatment, only $9(47.3 \%)$ patients had achieved normal levels prior to surgery. The rates of temporary and long-term hypoparathyroidism were 3 $(33.3 \%)$ and $1(11.1 \%)$, respectively, in the 9 patients with normal thyroid hormone levels versus 1 (10\%) and none, respectively, in the other 10 patients. No patient in the rapidly optimized group had documented evidence of significant voice change or post-operative vocal cord palsy and long-term recurrent laryngeal nerve damage. The number of patients who had achieved normal thyroid hormone levels pre-operatively was lower than that described in previous studies. This is likely due to higher baseline T4 and T3 in our patients (Table 2). Based on this, we suggest that a normal level need not be achieved as long as the levels are declining prior to surgery and the patients are clinically euthyroid.

In this series, the protocol for rapid optimization of poorly controlled group includes $0.3 \mathrm{mLs}$ Lugol's iodine three times a day in all patients at least 10 days prior to surgery and ATD if they have been tolerated. Cholestyramine, beta blockers and dexamethasone were added depending on patients' clinical response to initial medications. The effectiveness of protocols using different combinations such as those used in this study has not yet been reported in the literature.

This study is limited by its retrospective nature and some missing outcome data. Two patients in the "poorly controlled' group did not have severe biochemical hyperthyroidism, but were judged by the treating clinician to merit 'rapid optimization' and close monitoring. They were therefore considered to be in the 'poorly controlled' group. A number of patient-related (such as age, gender and comorbidity) and treatment-related variables (such as individual surgeon, trainee or surgeon performed, cumulative experience over time) may have influenced outcomes and were not adjusted for in multivariable analyses. The numbers in the second group were limited, and this reduces the power and increases the type II error rate of some of the statistical analyses comparing the outcomes in the two groups. There is a concern that 'hungry bone syndrome' may be a significant contributor to post-operative hypocalcaemia. In the absence of biochemical parameters including magnesium, phosphate and alkaline phosphate measurements after surgery, we are unable to distinguish clearly between hungry bone syndrome and 
hypoparathyroidism as causes of post-operative hypocalcaemia. However, within the constraints of the analysis presented, the observation of similar rates of 'low calcium' on the day after surgery argues against a significant impact of hungry bone syndrome in the rapidly optimized cohort of patients.

However, this study still provides evidence that rapid pre-optimization in poorly controlled GD and urgent surgery are effective and safe. The protocol for pre-operative optimization was left to the discretion of the treating clinicians. Based on this experience and following discussion of these results in a multi-disciplinary team, we have formulated a protocol for pre-operative rapid preparation in patients with Graves' disease who have not been adequately controlled in an elective, outpatient setting or those who need urgent surgical treatment of Graves' disease (such as those needing urgent treatment of other newly diagnosed conditions). In this protocol (Fig. 3), rapid preparation is started at least 10 days prior to the proposed operation with the objective of ensuring that the patient is clinically euthyroid and has free T4 levels of $<30 \mathrm{pmol} / \mathrm{L}$ and free T3 levels of $<10 \mathrm{pmol} / \mathrm{L}$ on the day of surgery. All patients are advised to continue their current anti-thyroid medications and commence Lugol's iodine $(0.3 \mathrm{~mL}$ tds) and propranolol (20 mg tds) 10 days prior to surgery. The biochemistry is monitored very closely as illustrated in Fig. 3 and further drugs (such as cholestyramine and dexamethasone) are added or the dose of medications are adjusted in accordance with the biochemical results. Other drugs such as lithium [30] and therapeutic plasmapheresis [31] have been reported as being effective in the rapid control of thyrotoxicosis prior to ablative treatment, but the authors do not have any experience of these modalities.

In summary, the rapid pre-operative treatment of patients with poorly controlled Graves' disease is effective, safe and there is no significant difference in morbidity compared to usual treatment.

Acknowledgements The authors wish to acknowledge all consultants and staff in the Department Of Endocrinology, Sheffield Teaching Hospitals, and the endocrine surgeons involved in the management of these patients.

Funding No external funding or grant was obtained.

\section{Compliance with ethical standards}

Conflict of interest The authors declare that they have no competing interests.

Open Access This article is distributed under the terms of the Creative Commons Attribution 4.0 International License (http://crea tivecommons.org/licenses/by/4.0/), which permits unrestricted use, distribution, and reproduction in any medium, provided you give appropriate credit to the original author(s) and the source, provide a link to the Creative Commons license, and indicate if changes were made.

\section{References}

1. Brent GA (2008) Clinical practice. Graves' disease. N Engl J Med 358:2594-2605

2. Hussain YS, Hookham JC, Allahabadia A et al (2017) Epidemiology, management and outcomes of Graves' disease-real life data. Endocrine 56:568-578

3. Abraham P, Avenell A, McGeoch SC et al (2010) Antithyroid drug regimen for treating Graves' hyperthyroidism. Cochrane Database Syst Rev 1:CD003420

4. Girgis CM, Champion BL, Wall JR (2011) Current concepts in graves' disease. Ther Adv Endocrinol Metab 2:135-144

5. Zambudio AR, Rodriguez J, Riquelme J et al (2004) Prospective study of postoperative complications after total thyroidectomy for multinodular goiters by surgeons with experience in endocrine surgery. Ann Surg 240:18-25

6. Langley RW, Burch HB (2003) Perioperative management of the thyrotoxic patient. Endocrinol Metab Clin North Am 32:519-534

7. Berghout A, Wiersinga WM, Brummelkamp WH (1989) Sodium ipodate in the preparation of Graves' hyperthyroid patients for thyroidectomy. Horm Res 31:256-260

8. Bogazzi F, Miccoli P, Berti P et al (2002) Preparation with iopanoic acid rapidly controls thyrotoxicosis in patients with amiodarone-induced thyrotoxicosis before thyroidectomy. Surgery 132:1114-1117 (discussion 1118)

9. Baeza A, Aguayo J, Barria M et al (1991) Rapid preoperative preparation in hyperthyroidism. Clin Endocrinol 35:439-442

10. Panzer C, Beazley R, Braverman L (2004) Rapid preoperative preparation for severe hyperthyroid Graves' disease. J Clin Endocrinol Metab 89:2142-2144

11. Fischli S, Lucchini B, Muller W et al (2016) Rapid preoperative blockage of thyroid hormone production/secretion in patients with Graves' disease. Swiss Med Wkly 146:w14243

12. Rubio GA, Koru-Sengul T, Vaghaiwalla TM et al (2017) Postoperative outcomes in Graves' Disease patients: results from the nationwide inpatient sample database. Thyroid 27:825-831

13. Pesce CE, Shiue Z, Tsai HL et al (2010) Postoperative hypocalcemia after thyroidectomy for Graves' disease. Thyroid 20:1279-1283

14. Chiang FY, Lin JC, Wu CW et al (2006) Morbidity after total thyroidectomy for benign thyroid disease: comparison of Graves' disease and non-Graves' disease. Kaohsiung J Med Sci 22:554-559

15. Hassan I, Danila R, Aljabri H et al (2008) Is rapid preparation for thyroidectomy in severe Graves' disease beneficial? The relationship between clinical and immunohistochemical aspects. Endocrine 33:189-195

16. Yamashita H, Murakami T, Noguchi S et al (1999) Postoperative tetany in Graves disease: important role of vitamin D metabolites. Ann Surg 229:237-245

17. Stedman T, Chew P, Truran P et al (2017) Modification, validation and implementation of a protocol for post-thyroidectomy hypocalcaemia. Ann R Coll Surg Engl 100:1-5

18. Akamizu T (2018) Thyroid storm: a Japanese perspective. Thyroid 28:32-40

19. Glinoer D, Hesch D, Lagasse R et al (1987) The management of hyperthyroidism due to Graves' disease in Europe in 1986. Results of an international survey. Acta Endocrinol Suppl (Copenh) 285:3-23 
20. Patwardhan NA, Moront M, Rao S et al (1993) Surgery still has a role in Graves' hyperthyroidism. Surgery 114:1108-1112 (discussion 1112-1103)

21. Hookham J, Collins EE, Allahabadia A et al (2017) Variation in the use of definitive treatment options in the management of Graves' disease: a UK clinician survey. Postgrad Med J 93:198-204

22. Stalberg P, Svensson A, Hessman O et al (2008) Surgical treatment of Graves' disease: evidence-based approach. World J Surg 32:1269-1277. https://doi.org/10.1007/s00268-008-9497-9

23. Ross DS, Burch HB, Cooper DS et al (2016) 2016 American thyroid association guidelines for diagnosis and management of hyperthyroidism and other causes of thyrotoxicosis. Thyroid 26:1343-1421

24. Palit TK, Miller CC 3rd, Miltenburg DM (2000) The efficacy of thyroidectomy for Graves' disease: a meta-analysis. J Surg Res 90:161-165

25. Hookham J, Truran P, Allahabadia A et al (2017) Patients' perceptions and views of surgery and radioiodine ablation in the definitive management of Graves' disease. Postgrad Med J 93:266-270
26. Gorman CA (1995) Therapeutic controversies. Radioiodine therapy does not aggravate Graves' ophthalmopathy. J Clin Endocrinol Metab 80:340-342

27. Tallstedt L, Lundell G, Torring O et al (1992) Occurrence of ophthalmopathy after treatment for Graves' hyperthyroidism. The Thyroid Study Group. N Engl J Med 326:1733-1738

28. Shinall MC Jr, Broome JT, Nookala R et al (2013) Total thyroidectomy for Graves' disease: compliance with American Thyroid Association guidelines may not always be necessary. Surgery 154:1009-1015

29. Al Jassim A, Wallace T, Bouhabel S et al (2018) A retrospective cohort study: do patients with graves' disease need to be euthyroid prior to surgery? J Otolaryngol Head Neck Surg 47:37

30. Nair GC, Balu MJC, Menon R et al (2018) Preoperative preparation of hyperthyroidism for thyroidectomy-role of supersaturated iodine and lithium carbonate. Indian J Endocrinol Metab 22:392-396

31. Simsir IY, Ozdemir M, Duman S et al (2018) Therapeutic plasmapheresis in thyrotoxic patients. Endocrine 62:144-148

Publisher's Note Springer Nature remains neutral with regard to jurisdictional claims in published maps and institutional affiliations. 